\title{
Effect of film thickness and dielectric environment on optical transmission through subwavelength holes
}

\author{
S. Carretero-Palacios, ${ }^{1}$ F. J. García-Vidal, ${ }^{2}$ L. Martín-Moreno, ${ }^{1}$ and Sergio G. Rodrigo ${ }^{1}$ \\ ${ }^{1}$ Instituto de Ciencia de Materiales de Aragón and Departamento de Física de la Materia Condensada, CSIC-Universidad de Zaragoza, \\ E-50009, Zaragoza, Spain \\ ${ }^{2}$ Departamento de Física Teórica de la Materia Condensada, Universidad Autónoma de Madrid, E-28049 Madrid, Spain
}

(Received 11 November 2011; revised manuscript received 12 December 2011; published 12 January 2012)

\begin{abstract}
We present a detailed theoretical study for the spectral position of transmission resonances appearing in isolated subwavelength apertures in metallic films. We provide analytical expressions for the resonant wavelength as a function of the film thickness and the dielectrics surrounding (and filling) the system that are valid for hole shapes supporting large-cutoff wavelengths and for both isolated and periodically arranged holes. Our results are quantitatively valid in the microwave and terahertz regimes, but they also have qualitative validity in the optical regime. Our results show that for unfilled holes, in the limiting case when the hole is in a very thin film (metal thickness much smaller than the wavelength), the transmission resonance is controlled by a length scale related to the vanishing of the effective admittance of vacuum, as seen from the hole. On the contrary, for metal thicknesses larger than half the wavelength, the transmission resonance is controlled by the cutoff of the fundamental waveguide mode inside the hole. When thin films and high-index dielectrics are combined, the spectral location of the maximum transmission can be strongly redshifted compared to the cutoff wavelength of the apertures, and transmission intensity is substantially enhanced.
\end{abstract}

DOI: 10.1103/PhysRevB.85.035417

PACS number(s): 41.20.Jb, 42.25.Bs, 42.79.Ag, 78.66.Bz

\section{INTRODUCTION}

During the last decades, there has been an increasing interest in transmission resonances through holes drilled in metal films. Broadly speaking, we can identify two kinds of resonances: those associated with extended surface modes and those identified with modes localized in the vicinity of individual holes.

Resonances based on extended modes appear in hole arrays and lead to the phenomenon of extraordinary optical transmission (EOT). ${ }^{1,2}$ For optically thick metal films (i.e., when $\delta<h<\lambda$, where $\delta$ is the skin depth, $h$ is the film thickness, and $\lambda$ is the incident wavelength) in a symmetric and uniform dielectric environment (with dielectric constant $\varepsilon$ ), these resonances emerge at $\lambda_{R} \approx \sqrt{\varepsilon} p$, with $p$ being the periodicity of the array. The transmission process has been described as a resonant interplay between surface-plasmon polaritons (SPPs) at each side of the metal surface evanescently coupled through the holes. ${ }^{3}$ Also EOT has been reported ${ }^{4,5}$ in optically thin metal films $(h \leqslant \delta)$, where transmission may occur through both the holes and the metal layer. In this case, the coupling of light with short-range surface plasmons redshifts the EOT peak to wavelengths larger than the periodicity of the array.

Localized resonances are essentially Fabry-Perot (FP) resonances. For symmetric dielectric configurations (and vacuum inside the apertures), the spectral location of the resonance appearing at the largest wavelength is $\lambda_{R} \approx \lambda_{c},{ }^{6-16}$ where $\lambda_{c}$ is the cutoff wavelength of the empty apertures. They have been attributed to the propagation of light through the fundamental waveguide mode (the least decaying one) inside the holes, and their appearance is related to the strong reduction of the propagation constant of such a mode close to $\lambda_{c}$. This implies that the field inside the holes does not experience a strong decay, photons spend a long time in the system, and transmission resonances can eventually take place. Recent numerical studies and experiments of near-field enhancement $^{17-19}$ and enhanced transmission ${ }^{20-22}$ have shown unexpected redshifts of the near field and transmission features compared to $\lambda_{c}$. These works consider either isolated holes or arrays of apertures combined with dielectric substrates and are developed in the terahertz $(\mathrm{THz})$ and optical regimes. In these works, a qualitative and phenomenological description of the unusual redshift of the localized resonance, based on the film thickness and the dielectric substrates, is presented. However, quantitative theory for the location of $\lambda_{R}$ is lacking. The fact that these transmission resonances appear in both hole arrays and single holes indicates that the electromagnetic (EM) modes responsible for the resonances are localized. Since they may appear beyond the cutoff wavelength, throughout this paper we will refer to this phenomenon as localized extraordinary optical transmission (LEOT).

Here we present a mathematical description of the LEOT phenomenon that provides analytical expressions of $\lambda_{R}$ as a function of the film thickness and the dielectric environment. We consider both symmetric dielectric configurations (where the dielectric constants of the cover, $\varepsilon_{1}$, and the substrate, $\varepsilon_{3}$, are the same) and asymmetric ones $\left(\varepsilon_{1} \neq \varepsilon_{3}\right)$ for any film thickness. We also reveal that different combinations of the dielectrics outside and inside the apertures $\left(\varepsilon_{2}\right)$ may blueshift or redshift the transmission resonance compared to that of a homogeneous system (i.e., $\varepsilon_{1}=\varepsilon_{2}=\varepsilon_{3}$ ). We demonstrate that the spectral position of the maximum transmittance is controlled by a length scale that characterizes the coupling of the hole with radiation $\left(\lambda_{0}\right)$.

Our theoretical approach is valid for any hole shape, provided it fulfils the condition $\sqrt{\varepsilon_{2}} \lambda_{c}>\sqrt{A}$, where $A$ is the "hole area." Examples of these "large-cutoff holes" are rectangular and annular holes with a large aspect ratio or apertures of any shape filled ${ }^{23}$ with a sufficiently large $\varepsilon_{2}$. In particular, to illustrate our findings we consider both 
rectangular and annular holes, and we discuss the differences with empty circular apertures (which do not support largecutoff wavelengths). By comparing with finite-difference time domain (FDTD) numerical results, we show that our results are virtually exact in the $\mathrm{THz}$ and microwave regimes and qualitatively valid in the optical regime.

This paper is organized as follows: In Sec. II we describe the theoretical method used, the coupled-mode method (CMM). In Sec. III we study the location of maximum transmission of an isolated aperture in a symmetric dielectric configuration, and the differences between empty circular apertures and annular holes are also discussed. Asymmetric systems are considered in Sec. IV, and the extension of our results to hole arrays and the optical regime appears in Sec. V. Finally, conclusions are presented in Sec. VI.

\section{COUPLED-MODE METHOD}

To get physical insight into the origin of the strong redshift of $\lambda_{R}$ found when opaque thin films $(\delta<<h<<\lambda)$ and highindex dielectrics are combined, here we summarize the main ingredients and expressions of the theoretical formalism used, the coupled-mode method. We refer to Refs. 24 and 2 for details and derivations.

We take the metal as a perfect electrical conductor (PEC) (i.e., the dielectric constant of the metal is $\left|\varepsilon_{m}\right|=\infty$ ), which is a good approximation for metals in the $\mathrm{THz}$ regime, and illumination at normal incidence (the electric field points parallel to the $x$ direction; see schematics in Fig. 1). Within the CMM, space is divided into three different regions: the illuminated region (I), the holey-metal region (II), and the transmission region (III). Electromagnetic fields are expanded into the proper eigenmodes in each one: plane waves in regions I and III and waveguide modes inside the holes (region II). More precisely, plane waves are characterized by the in-plane component of the wave vector $\mathbf{k}=\left(k_{x}, k_{y}\right)$ (the $z$ component of the wave vector satisfies $k_{z}=\sqrt{\varepsilon_{1,3} g^{2}-k^{2}}$, with $\left.g=2 \pi / \lambda\right)$ and the polarization, $\sigma=p$ or $s$.

Within the PEC approximation, the EM modes inside the apertures coincide with the waveguide modes of those apertures, which are known analytically for some geometries (see Refs. 25 and 26 for further details). In the case of subwavelength holes, considering only the least decaying mode inside the apertures provides a good approximation of transmission properties. . 26,27

Imposing the appropriate matching conditions at the interfaces (I-II and II-III), we obtain a coupled system of equations

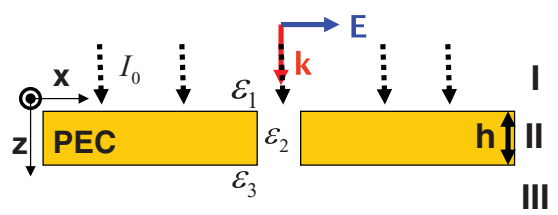

FIG. 1. (Color online) Diagram of the systems under study with an arbitrary hole aperture perforated on a metal film of thickness $h$. The structure is illuminated by a $p$-polarized plane wave at normal incidence. We consider semi-infinite dielectrics at illumination and transmission regions, with dielectric constants $\varepsilon_{1}$ and $\varepsilon_{3}$, respectively, and a dielectric constant $\varepsilon_{2}$ filling the holes. for the modal amplitudes of the electric field at the entrance $(E)$ and exit $\left(E^{\prime}\right)$ sides of the cavities:

$$
\begin{aligned}
\left(G^{I}-\Sigma\right) E-G_{\nu} E^{\prime} & =I_{0}, \\
\left(G^{I I I}-\Sigma\right) E^{\prime}-G_{\nu} E & =0,
\end{aligned}
$$

whose solution is given by

$$
\begin{aligned}
E & =\frac{\left(G^{I I I}-\Sigma\right)}{\left(G^{+}-\Sigma\right)^{2}-G_{v}^{2}-\left(G^{-}\right)^{2}} I_{0}, \\
E^{\prime} & =\frac{G_{v}}{\left(G^{+}-\Sigma\right)^{2}-G_{v}^{2}-\left(G^{-}\right)^{2}} I_{0} .
\end{aligned}
$$

In the above expressions, $I_{0}$ accounts for the external illumination impinging directly on the holes, and it measures the overlap between the incident plane wave and the fundamental mode inside the hole, $\Sigma$ represents the light that comes back to the aperture after bouncing back and forth inside the cavities, and the term $G_{v}$ is linked to the coupling of EM fields at the two sides of the film through the holes. It is important to remark that both $\Sigma$ and $G_{v}$ depend on $h$ and the propagation constant inside the apertures $\beta$ as

$$
\begin{aligned}
\Sigma & =i \frac{\beta}{g} \frac{e^{i \beta h}+e^{-i \beta h}}{e^{i \beta h}-e^{-i \beta h}}, \\
G_{\nu} & =2 i \frac{\beta}{g} \frac{e^{i \beta h}}{e^{2 i \beta h}-1} .
\end{aligned}
$$

Also, in Eqs. (1) and (2), Green's functions $G^{I}\left(\lambda ; \varepsilon_{1}\right)$ and $G^{I I I}\left(\lambda ; \varepsilon_{3}\right)$ represent effective admittances (in regions I and III, respectively) that account for the EM coupling of the aperture and the external radiation as seen by the holes, and we have defined

$$
\begin{aligned}
G^{+} & =\left[G^{I}\left(\lambda ; \varepsilon_{1}\right)+G^{I I I}\left(\lambda ; \varepsilon_{3}\right)\right] / 2, \\
G^{-} & =\left[G^{I}\left(\lambda ; \varepsilon_{1}\right)-G^{I I I}\left(\lambda ; \varepsilon_{3}\right)\right] / 2 .
\end{aligned}
$$

The general expression for hole arrays can be written as $G^{I}=i \sum_{\mathbf{k} \sigma} Y_{\mathbf{k} \sigma}^{I}\left|S_{\mathbf{k} \sigma}\right|^{2}$, where $Y_{\mathbf{k} s}^{I}=k_{z} / g$ and $Y_{\mathbf{k} p}^{I}=\varepsilon_{1} g / k_{z}$ (and the same for $G^{I I I}$ with $\varepsilon_{3}$ ). The overlaps are defined as an integral over the hole shape

$$
S_{\mathbf{k} \sigma}=\int d r_{\|} \vec{V}_{\sigma} \cdot \vec{E}_{\|}\left(r_{\|}\right) \cdot e^{i \vec{k}_{\|} \cdot \vec{r}_{\|}},
$$

with the bivectors $\vec{V}_{p}=\left(k_{x}, k_{y}\right) / k_{\|}, \vec{V}_{s}=\left(-k_{y}, k_{x}\right) / k_{\|}$and $k_{\|}^{2}=k_{x}^{2}+k_{y}^{2}$. In the expressions of $G^{I, I I I}$ for hole arrays, $\mathbf{k}$ runs over the reciprocal lattice vectors, whereas for isolated holes the sum over $\mathbf{k}$ must be substituted by an integral $\sum_{\mathbf{k}} \rightarrow$ $1 /(2 \pi)^{2} \int d \mathbf{k}$.

Note that the imaginary part of the Green's function $G_{i}$ controls the coupling with radiative modes, while the real part $G_{r}$ is related to the coupling of the aperture with evanescent modes.

An important property of $G^{I, I I I}$, which will be used later on, is how it transforms under changes of the dielectric constant: $:^{27}$

$$
G^{I}\left(\sqrt{\varepsilon_{1}} \lambda ; \varepsilon_{1}\right)=\sqrt{\varepsilon_{1}} G^{I}(\lambda ; 1)
$$

and the same for $G^{I I I}$ with $\varepsilon_{3}$. 
Finally, transmission can be written in terms of the modal amplitudes of the electric field as ${ }^{12,27,28}$

$$
T=\frac{1}{\sqrt{\varepsilon_{1}}} \operatorname{Im}\left(G_{\nu} E^{*} E^{\prime}\right)=\frac{1}{\sqrt{\varepsilon_{1}}} G_{i}^{I I I}\left|E^{\prime}\right|^{2} .
$$

\section{SYMMETRIC CONFIGURATIONS, $\varepsilon_{1}=\varepsilon_{3}$}

Since the LEOT phenomenon is found in both hole arrays and single holes, for simplicity we will concentrate on the study of isolated apertures, where the lattice resonances are not present.

Let us first investigate the spectral location of maximum transmission in symmetric configurations $\left(\varepsilon_{1}=\varepsilon_{3}=\varepsilon\right)$. In this case, $G^{I}\left(\lambda ; \varepsilon_{1}\right)=G^{I I I}\left(\lambda ; \varepsilon_{3}\right)=G(\lambda ; \varepsilon)$ and $G^{-}=0$, so after factoring $E$ and $E^{\prime}$ in Eq. (2) and substituting their expression in Eq. (7), transmission becomes

$$
T=\frac{\left|I_{0}\right|^{2}}{\sqrt{\varepsilon}} \frac{G_{i}\left|G_{\nu}\right|^{2}}{\left[|G-\Sigma|^{2}-\left|G_{\nu}\right|^{2}\right]^{2}+4 G_{i}^{2}\left|G_{\nu}\right|^{2}} .
$$

As we said in the Introduction, transmission maxima associated with FP modes appear when the EM energy at the entrance and exit sides of the apertures are equal, ${ }^{6-16}$ i.e., $|E|=\left|E^{\prime}\right|$, which occurs for $|G-\Sigma|=\left|G_{v}\right|$. If we now replace the condition $|G-\Sigma|=\left|G_{\nu}\right|$ in Eq. (8), we retrieve the known expression for the maximum transmittance at maximum: ${ }^{12}$

$$
T_{R}=\frac{\left|I_{0}\right|^{2}}{4 \sqrt{\varepsilon}} \frac{1}{G_{i}} .
$$

Interestingly, $G_{i}$ controls the intensity of transmission resonances, whereas the condition for the location of the resonance is governed by $G_{r}$, and it can be written as ${ }^{12}$

$$
2 G_{r}=\frac{|G|^{2}-Y_{T E}^{2}}{Y_{T E}} \tan (\beta h),
$$

with $Y_{T E}=\beta / g$.

For $G_{r} \ll|G|$, Eq. (10) has a solution for $\tan (\beta h) \approx 0$. Previous works ${ }^{12}$ analyzed the specific case $h \leqslant \lambda$ and $\varepsilon=1$, and it was found that resonances occurred around $\beta=0$, i.e., close to $\lambda_{c}$. These localized resonances are FP resonances modified by the presence of openings, so $\beta h \approx 0$ corresponds to the zeroth-order FP.

Next, we will extend this previous work and will analyze the whole dependance with the metal thickness and the dielectric environment.

Although Eq. (2) can be solved exactly, in order to get deeper physical insight it is convenient to define the following change of variables, which allows us to describe the transmission process into two well-defined transmission channels:

$$
\begin{aligned}
& E_{S}=\frac{1}{\sqrt{2}}\left(E-E^{\prime}\right), \\
& E_{A}=\frac{1}{\sqrt{2}}\left(E+E^{\prime}\right) .
\end{aligned}
$$

Then, Eq. (1) can be written as an equivalent system of uncoupled equations in terms of a symmetric $\left(E_{S}\right)$ transmission channel, where the electric fields at the entrance and at the exit of the apertures point in the same direction $\left(E=-E^{\prime}\right),{ }^{29}$ and an antisymmetric $\left(E_{A}\right)$ channel, where the electric fields point in opposite directions $\left(E=E^{\prime}\right)$ :

$$
\begin{aligned}
& \left(G+Y_{S}\right) E_{S}=\tilde{I}, \\
& \left(G+Y_{A}\right) E_{A}=\tilde{I} .
\end{aligned}
$$

Here $Y_{S} \equiv-\left(\Sigma-G_{\nu}\right)$ and $Y_{A} \equiv-\left(\Sigma+G_{\nu}\right)$ are effective admittances, and $\tilde{I}=I / \sqrt{2}$.

Within the new formulation, the resonant condition $(\beta h \approx$ $0)$ provides $Y_{A} \rightarrow \infty$. This means that, except in "extraordinary" cases (i.e., for PEC, close to the Rayleigh wavelength, when $G \rightarrow \infty), E_{A}$ is not excited. Therefore, in the limit of very thin films $(h \rightarrow 0)$, only the symmetric transmission channel $E_{S}$ remains, whereas the asymmetric channel $E_{A} \rightarrow 0$.

This is easy to understand because, since the asymmetric channel is characterized by electric fields at the entrance and exit sides of the apertures pointing in opposite directions, in thin films, there is not enough optical path for the field to flip the direction. As a result, for thin-metal films, only the symmetric channel is excited, and transmission can be written as

$$
T_{\text {approx }}=\frac{\left|I_{0}\right|^{2}}{4 \sqrt{\varepsilon}} \frac{G_{i}}{\left|G+Y_{S}\right|^{2}},
$$

with $Y_{S}=\frac{\beta^{2} h}{2 g}$.

Resonances in transmission will be given by minima in the denominator of Eq. (13). Because $G_{r}>>G_{i}$ and $\operatorname{Im}\left(Y_{S}\right)=0$ around the cutoff wavelength, zeros in the denominator occur at $\operatorname{Re}\left(G+Y_{S}\right)=0$.

The propagation constant is $\beta=\sqrt{\varepsilon_{2} g^{2}-g_{c}^{2}}$. Replacing this value in $Y_{S}$ and using Eq. (6), we obtain a transcendental equation for the resonant wavelength $\left[\lambda_{R}^{(a)}=2 \pi / g_{R}^{(a)}\right.$, where the upper index $(a)$ stands for approximation] that can be solved graphically,

$$
-G_{r}\left(\lambda_{R}^{(a)} ; \varepsilon\right)=\frac{2 \pi h}{\lambda_{c}^{2}}\left(\sqrt{\varepsilon_{2}} \lambda_{c}-\lambda_{R}^{(a)}\right) .
$$

This is one of the most important results we present here, and it describes the location of transmission resonances for any film thickness in a symmetric configuration and for any hole shape supporting large-cutoff wavelengths. Note that in the above expression, $\lambda_{c}$ is the cutoff wavelength of an empty hole $\left(\varepsilon_{2}=1\right)$, and we have considered that, close to the cutoff, $\beta \approx 2 g_{c}\left(\sqrt{\varepsilon_{2}} g_{R}-g_{c}\right)$.

An essential ingredient entering the condition for resonances in Eq. (14) appears on the left-hand side of the equation, i.e., $G_{r}$. This function can take either negative or positive values, and the sign and values of $G_{r}$ depend on the relative contribution of $p$-polarized (which is negative and dominates at $\lambda \rightarrow 0$ ) and $s$-polarized (which is positive and dominates at $\lambda \rightarrow \infty$ ) waves. Therefore, there exists a wavelength where the two contributions cancel each other, and we define this wavelength as $G_{r}\left(\lambda_{0} ; \varepsilon=1\right)=0$. If we add a dielectric constant different from unity in the cover and substrate, using Eq. (6), we find that the real part of the effective admittance cancels at $G_{r}\left(\sqrt{\varepsilon} \lambda_{0} ; \varepsilon\right)=0$. Note that $\lambda_{0}(\varepsilon)=\sqrt{\varepsilon} \lambda_{0}(\varepsilon=1)$. 


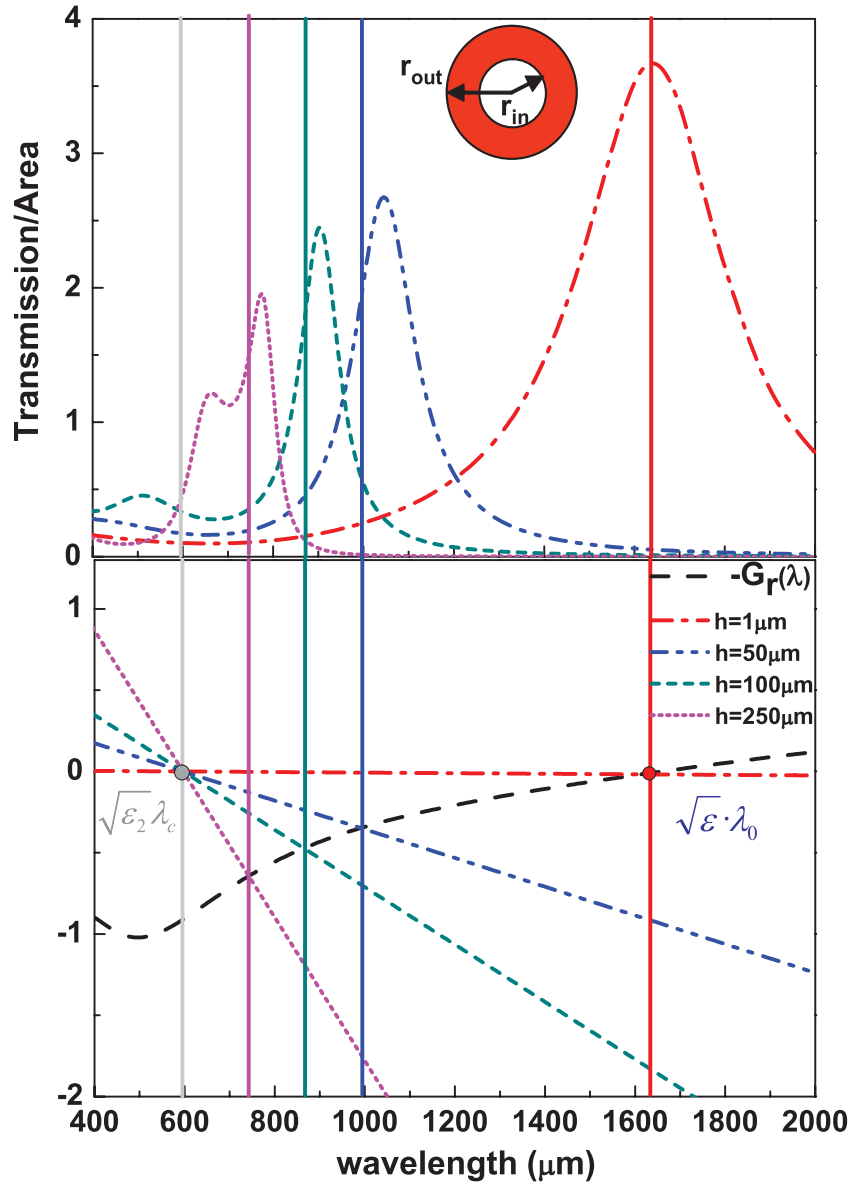

FIG. 2. (Color online) (top) Transmission spectra (normalized to $\left.\pi r_{\text {out }}^{2}\right)$ of an isolated annular hole $\left(r_{\text {in }}=90 \mu \mathrm{m}, r_{\text {out }}=100 \mu \mathrm{m}\right)$ drilled in a PEC of different film thicknesses $h$ (see labels) in a symmetric configuration with $\varepsilon=9$ and $\varepsilon_{2}=1$. (bottom) For the same system as that in the top panel, the dashed line depicts $-G_{r}$ as a function of the incident wavelength. Straight lines correspond to the right-hand side of Eq. (14) for each $h$ value. Vertical lines depict $\lambda_{R}^{(a)}$ in each case.

The right-hand side of Eq. (14) represents straight lines whose slopes are given by the film thickness $h$. According to these equations, the intersection of these straight lines with $-G_{r}$ gives the position of the resonant wavelengths $\lambda_{R}^{(a)}$ for each $h$ value.

As an illustration of this graphical solution, in Fig. 2 we consider a system with a single annular hole drilled in a PEC. Since we are illuminating the system at normal incidence, only the fundamental $\mathrm{TE}_{11}$ mode is considered inside the hole (annular holes support a TEM mode deprived of cutoff that is not excited at normal incidence ${ }^{30-32}$ ). The geometrical parameters are the following: inner radius $r_{\text {in }}=90 \mu \mathrm{m}$, outer radius $r_{\text {out }}=100 \mu \mathrm{m}$ [which provides a cutoff wavelength ${ }^{31,32}$ $\left.\lambda_{c} \approx \pi\left(r_{\text {in }}+r_{\text {out }}\right)=596 \mu \mathrm{m}\right]$, and we have taken $\varepsilon=9$, $\varepsilon_{2}=1$. The bottom panel in Fig. 2 shows the graphical solution of Eq. (14) for different $h$ values, and the top panel shows the corresponding transmission spectra normalized to $\pi r_{\text {out }}^{2}$. The top panel shows that in the regime $h \geqslant \lambda / 2$ (in this case, $h=250 \mu \mathrm{m})$ the resonances associated with the symmetric channel $E_{S}$ and with the asymmetric one $E_{A}$ emerge. In the graphical solution of Eq. (14) we observe that when the slope of the straight line tends to infinity $(h \rightarrow \infty)$, the intersection with $-G_{r}$ occurs at $\lambda_{R}^{(a)}=\sqrt{\varepsilon_{2}} \lambda_{c}$.

In contrast, in the limit of very thin films $(h<<\lambda)$, only $E_{S}$ can be excited, and for each $h$ value, there is just one transmission peak strongly redshifted compared to $\sqrt{\varepsilon_{2}} \lambda_{c}$. In this limit, the slope of the straight line tends to zero $(h \rightarrow 0)$ and $G_{r}\left(\lambda_{R}^{(a)}\right)=0$, then having $\lambda_{R}^{(a)}=\sqrt{\varepsilon} \lambda_{0}$.

Results in Fig. 2 show that Eq. (14) accurately provides the resonant wavelength for any film thickness in a symmetric dielectric environment, and the analytical expressions in the limit of thin and thick films are

$$
\begin{aligned}
& \lambda_{R}^{(a)}=\sqrt{\varepsilon} \lambda_{0}(h<<\lambda), \\
& \lambda_{R}^{(a)}=\sqrt{\varepsilon_{2}} \lambda_{c}(h \geqslant \lambda / 2) .
\end{aligned}
$$

Notice that Eqs. (15) reflect what could be intuitively expected: for thick films the resonance is governed by the properties of the waveguide (represented by $\lambda_{c}$ ), while for very thin films it is governed by the coupling of the hole to radiative regions (characterized by $\lambda_{0}$ ).

According to Eqs. (15), the resonant wavelength is larger than the cutoff wavelength for $\varepsilon>\varepsilon_{2}$ (or smaller for $\varepsilon<\varepsilon_{2}$ ). This result will be shown in Sec. V.

The limit $\lambda_{R} \approx \sqrt{\varepsilon} \lambda_{c}$ for thin films has been advanced in previous works when studying, both experimentally and theoretically, systems containing empty circular, square, and rectangular holes ${ }^{17,20,21}$ in asymmetric configurations. However, when rectangular holes combined with high-index dielectric substrates are considered, ${ }^{21}$ this prediction is not accurate enough, as has also been stated in Ref. 21. The proper description for $\lambda_{R}$ in asymmetric systems will be presented in Sec. IV.

To further illustrate the validity of both Eqs. (14) and (15), Fig. 3(a) shows the resonant wavelength of the symmetric mode obtained through the analytical approximation $\lambda_{R}^{(a)}$ and the exact position of the maximum transmission $\lambda_{R}$ calculated with the CMM, as a function of $h$, for an isolated rectangular hole $\left(a_{x}=10 \mu \mathrm{m}, a_{y}=350 \mu \mathrm{m}\right)$, with $\varepsilon=12$ and $\varepsilon_{2}=1$. The agreement of the two curves along with the limits predicted by Eqs. (15) for thin and thick films confirms our analytical results. The corresponding transmission intensities (normalized to the hole area) at $\lambda_{R}$ appear in Fig. 3(b).

The redshift observed in the LEOT phenomenon strongly influences transmission intensities at $\lambda_{R}$. It is not easy to find a more compact and accurate expression for $T_{R}$ that is valid for any hole shape than that given by Eq. (9) since the illumination term depends on the overlapping between the incident plane wave and the fundamental mode inside the hole and therefore on the hole shape. However, it is possible to find a general trend to know if the maximum intensity will increase or decrease with $\varepsilon$ and $h$. If we take into account (i) the Green's-function property given by Eq. (6), (ii) $G_{i}(\lambda ; 1) \propto$ $A / \lambda^{2}$ for subwavelength holes, and (iii) $\left|I_{0}(\varepsilon)\right| \propto \sqrt{\varepsilon}$, we find

$$
T_{R}\left(\lambda_{R} ; \varepsilon, \varepsilon_{2}\right) \propto \frac{\lambda_{R}^{2}}{\varepsilon} .
$$

Interestingly, for thin films $T_{R} \propto \lambda_{c}^{2}$, while for thick films $T_{R} \propto\left(\varepsilon_{2} / \varepsilon\right) \lambda_{c}^{2}$. This means that, for a given hole shape when the metal film is thin $(h<<\lambda)$, the peak location depends 


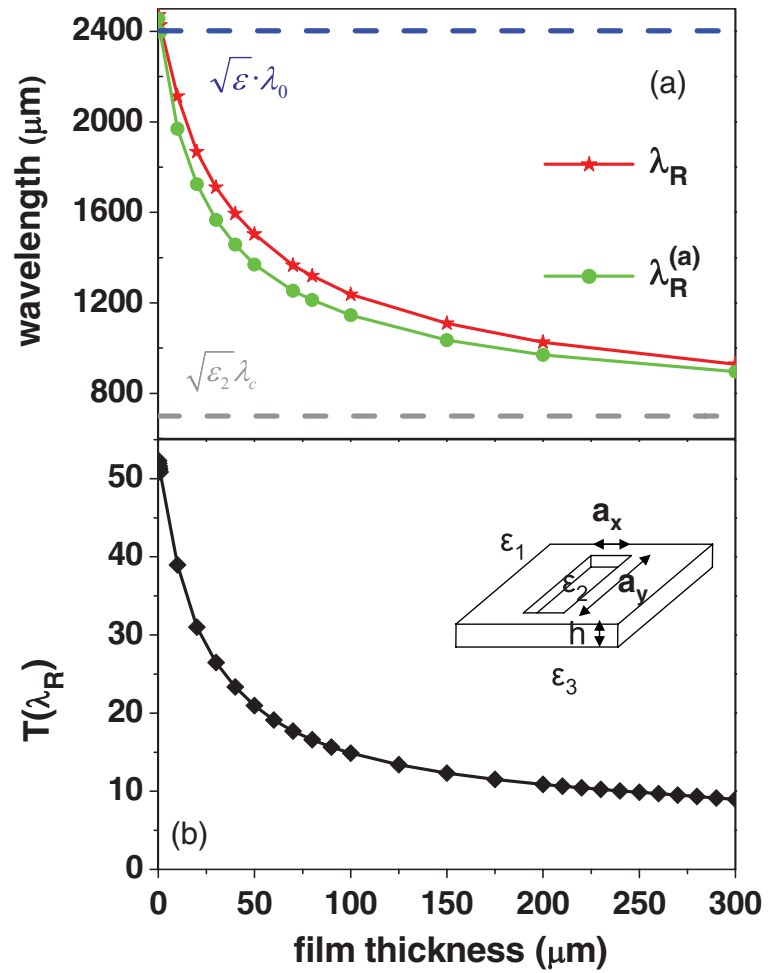

FIG. 3. (Color online) (a) Spectral wavelength of the transmission resonance as a function of the film thickness of an isolated rectangular hole $\left(a_{x}=10 \mu \mathrm{m}, a_{y}=350 \mu \mathrm{m}\right)$ with $\varepsilon=12$ and $\varepsilon_{2}=1$. Stars depict $\lambda_{R}$, and circles depict $\lambda_{R}^{(a)}$ obtained through the analytical approximation given by Eq. (14). (b) Corresponding transmission intensities (normalized to the hole area) at $\lambda_{R}$.

on the dielectric environment, but its intensity $T_{R}$ does not. In contrast, for the same hole shape but when $h \geqslant \lambda / 2$, the resonant wavelength does not depend on $\varepsilon$, but the intensity will be modulated by the factor $\varepsilon_{2} / \varepsilon$. Only for $\varepsilon_{2}>\varepsilon$ do holes in thick films transmit better than in thin ones. In Fig. 3(b), $\varepsilon_{2}<\varepsilon$, so in this case holey thin films transmit better than thick ones.

Furthermore, taking advantage of the property given by Eq. (6), it is easy to demonstrate that, in the limit of thin films,

$$
T\left(\lambda ; \varepsilon_{2}, 1\right)=T\left(\sqrt{\varepsilon} \lambda ; \varepsilon_{2}, \varepsilon\right) \quad(h \rightarrow 0) .
$$

So far, the analysis has dealt with structures that fulfill the condition $\sqrt{\varepsilon_{2}} \lambda_{c}>\sqrt{A}$. Let us now discuss the validity of the previous description for systems that do not fulfill that condition, such as empty circles. As already mentioned, resonant behavior in the subwavelength regime is related to small values of $G_{i}$, where photons stay for a long time in the system before coupling to radiative modes. This behavior is found in rectangles or annular holes. In contrast, for empty square or circular holes of the same area, radiation losses are larger, which also provides larger $G_{i}$ values, making the condition $G_{r}>>G_{i}$ less valid. Therefore, $G_{i}$ affects the spectral wavelength where the maximum appears, its intensity, and also its spectral width. Concerning $\lambda_{R}$ and the corresponding intensities $T_{R}$, Fig. 4 shows the transition from circles to annular holes of high aspect ratio. The geometrical parameters are chosen following the experimental ones for circular holes in Ref. 18 (see caption). The top panel shows

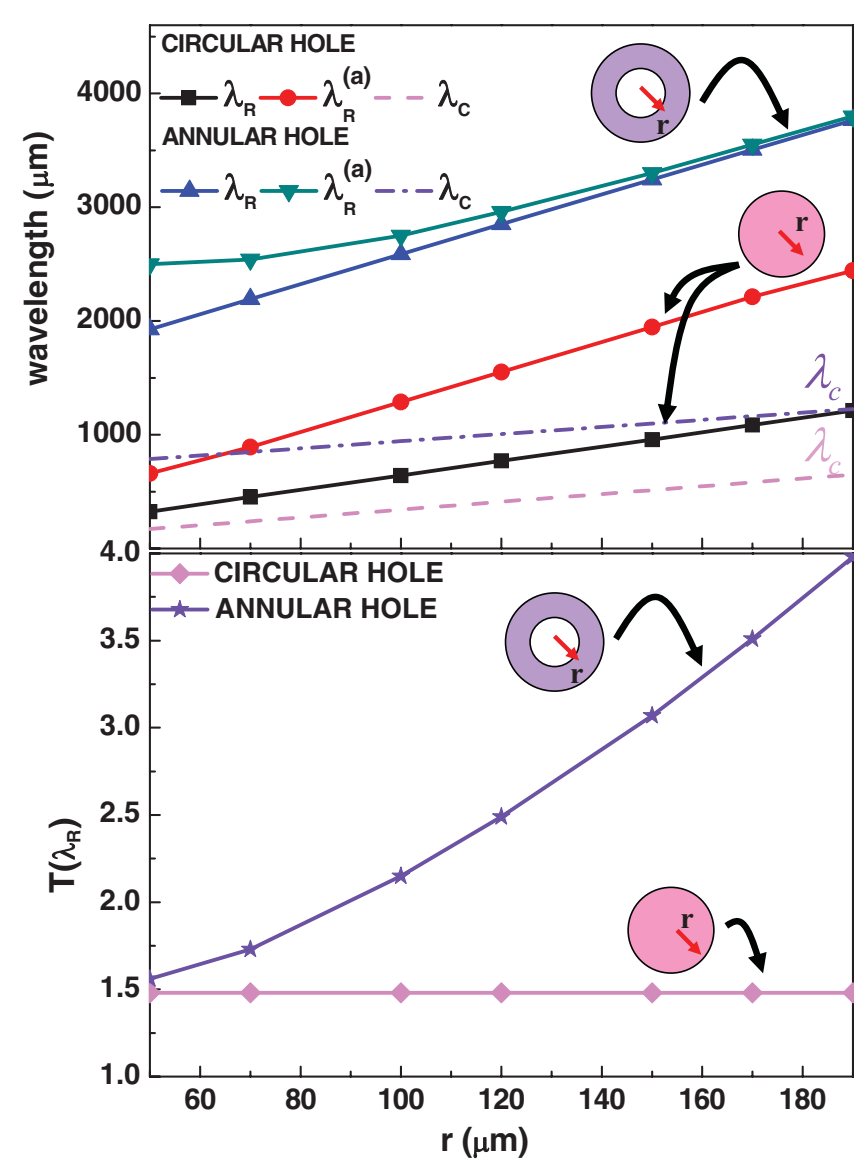

FIG. 4. (Color online) (top) Spectral wavelength of the transmission resonance as a function of $r$, where $r$ accounts for either the circular radius or $r_{\text {in }}$ in an annular hole with $r_{\text {out }}=200 \mu \mathrm{m}$. Dashed lines represent $\lambda_{c}$ in each case (see labels). The rest of parameters are $\varepsilon=11, \varepsilon_{2}=1$, and $h=0.2 \mu \mathrm{m}$. (bottom) Corresponding intensities (normalized to $\pi r^{2}$ ) at $\lambda_{R}$.

$\lambda_{R}, \lambda_{R}^{(a)}$, and $\lambda_{c}$ as a function of $r$ (which accounts for either the circular radius or $r_{\text {in }}$ in an annular hole with $r_{\text {out }}=200 \mu \mathrm{m}$ ). Although in all cases there is a redshift of $\lambda_{R}$ compared to $\lambda_{c}$, our theoretical prediction is only valid for annular holes. Note also that our approximation applies for very small apertures, which fulfill the condition ${ }^{12} G_{i} \approx 0$. The bottom panel shows the corresponding intensities at $\lambda_{R}$, where we can observe that the larger $\lambda_{R}$ is, the larger $T_{R}$ normalized to the hole area is.

\section{ASYMMETRIC CONFIGURATIONS, $\varepsilon_{1} \neq \varepsilon_{3}$}

Next, we will consider asymmetric configurations, where $\varepsilon_{1} \neq \varepsilon_{3}$. We will follow the same reasoning as in Sec. III.

Within this new dielectric configuration, we can solve again Eq. (1) in order to find analytical expressions for $\lambda_{R}$. In all cases analyzed, we have found that $G^{-}<<G^{+}$even when the dielectric constants of each region are very dissimilar. After some algebra, we obtain an analogous expression to that given by Eq. (8) for the transmission of light:

$$
T \approx \frac{\left|I_{0}\right|^{2}}{\sqrt{\varepsilon_{1}}} \frac{G_{i}^{I I I}\left|G_{\nu}\right|^{2}}{\left[\left|G^{+}-\Sigma\right|^{2}-\left|G_{\nu}\right|^{2}\right]^{2}+4\left(G_{i}^{+}\right)^{2}\left|G_{\nu}\right|^{2}} .
$$




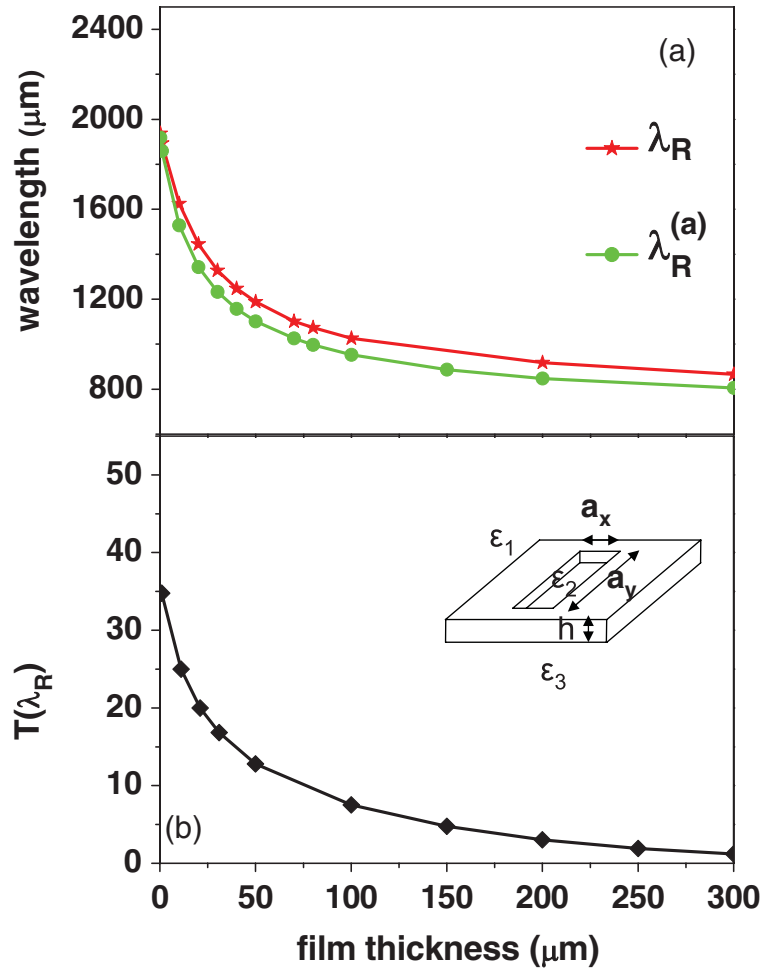

FIG. 5. (Color online) Same calculations as those in Fig. 3 with $\varepsilon_{1}=\varepsilon_{2}=1$ and $\varepsilon_{3}=12$, considering Eq. (20).

In the limit of thin films, this expression simplifies to

$$
T_{\text {approx }} \approx \frac{\left|I_{0}\right|^{2}}{4 \sqrt{\varepsilon_{1}}} \frac{G_{i}^{I I}}{\left|G^{+}+Y_{S}\right|^{2}},
$$

and the location of the resonant wavelength is given by

$$
-G_{r}^{+}\left(\lambda_{R}^{(a)} ; \varepsilon_{1} ; \varepsilon_{3}\right)=\frac{2 \pi h}{\lambda_{c}^{2}}\left(\sqrt{\varepsilon_{2}} \lambda_{c}-\lambda_{R}^{(a)} .\right)
$$

The last equation can also be solved graphically to get the peak location as a function of the film thickness. Figure 5 shows the same calculations as those in Fig. 3, but with $\varepsilon_{1}=\varepsilon_{2}=1$ and $\varepsilon_{3}=12$. Again, the resonant wavelength obtained through Eq. (20) and the exact result provided by the CMM agree very well.

Nevertheless, it is interesting to get analytical expressions for $\lambda_{R}$ in the limits of thin and thick films, as we got for symmetric configurations. Clearly, for thick enough films, the resonant wavelength is also given by $\lambda_{R}=\sqrt{\varepsilon_{2}} \lambda_{c}$ because when $h \rightarrow \infty$, the intersection of $G^{+}$and the straight line of infinite slope occurs at $\sqrt{\varepsilon_{2}} \lambda_{c}$.

For thin films, the resonant wavelength is given by the condition $G_{r}^{+}\left(\lambda_{R}^{(a)} ; \varepsilon_{1} ; \varepsilon_{3}\right)=0$. The derivation presented here justifies mathematically the numerical results reported in Ref. 21. Figure 6 shows $\lambda_{R}$, together with $\lambda_{R}^{(a)}$ [defined through $\left.G_{r}^{+}\left(\lambda_{R}^{(a)}\right)=0\right]$ in a rectangular hole, with $\varepsilon_{1}=\varepsilon_{2}=1$, drilled in a thin film of $h=1 \mu \mathrm{m}$, as a function of the dielectric constant of the substrate $\varepsilon_{3}$. Clearly, $\lambda_{R}^{(a)}$ marks the wavelength for maximum transmission.

However, getting an analytical expression of $\lambda_{R}^{(a)}$ is not that easy. The main problem arises because $G_{r}^{+}$does not transform

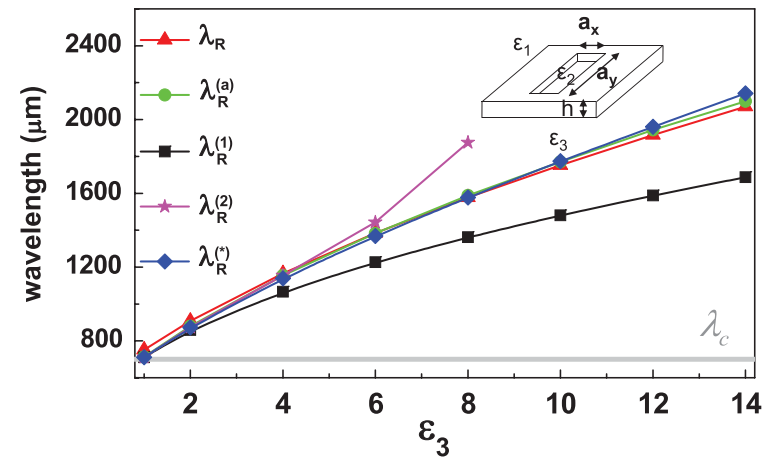

FIG. 6. (Color online) Position of transmission maximum as a function of $\varepsilon_{3}$ in an isolated rectangular hole $\left(a_{x}=10 \mu \mathrm{m}, a_{y}=350\right.$ $\mu \mathrm{m})$ with $h=1 \mu \mathrm{m}$ and $\varepsilon_{1}=\varepsilon_{2}=1$. Triangles correspond to $\lambda_{R}$, circles correspond to $\lambda_{R}^{(a)}$ obtained through $G_{r}^{+}\left(\lambda_{R}^{(a)}\right)=0$, squares depict $\lambda_{R}^{(1)}$ obtained through Eq. (23), stars correspond to $\lambda_{R}^{(2)}$ given by Eq. (24), and diamonds depict $\lambda_{R}^{(*)}$ obtained through Eq. (25).

with $\varepsilon$ as $G_{r}$ does in symmetric configurations, and instead, it transforms as follows:

$$
\begin{aligned}
& G^{+}\left(\lambda ; \varepsilon_{1} ; \varepsilon_{3}\right) \\
& \quad=\frac{1}{2}\left[\sqrt{\varepsilon_{1}} G^{I}\left(\frac{\lambda}{\sqrt{\varepsilon_{1}}} ; 1\right)+\sqrt{\varepsilon_{3}} G^{I I I}\left(\frac{\lambda}{\sqrt{\varepsilon_{3}}} ; 1\right)\right] .
\end{aligned}
$$

In general, when $G^{+}\left(\lambda_{R}^{(a)} ; \varepsilon_{1} ; \varepsilon_{3}\right)=0$, neither $G^{I}$ nor $G^{I I I}$ are zero, so in order to solve Eq. (21) we need the whole dependance of $G^{I}$ and $G^{I I I}$ with $\lambda$.

Fortunately, from the above property we can still obtain approximate explicit expressions for $\lambda_{R}^{(a)}$. To do that, we first approximate linearly both $G^{I}$ and $G^{I I I}$ around $\lambda_{0}$ as $G_{r}(\lambda ; 1) \approx A\left(\lambda-\lambda_{0}\right)$; that is,

$$
\begin{aligned}
& G^{+}\left(\lambda ; \varepsilon_{1} ; \varepsilon_{3}\right) \\
& \quad=\frac{A}{2}\left[\sqrt{\varepsilon_{1}}\left(\frac{\lambda}{\sqrt{\varepsilon_{1}}}-\lambda_{0}\right)+\sqrt{\varepsilon_{3}}\left(\frac{\lambda}{\sqrt{\varepsilon_{3}}}-\lambda_{0}\right)\right] .
\end{aligned}
$$

Applying $G^{+}\left(\lambda_{R}^{(a)} ; \varepsilon_{1} ; \varepsilon_{3}\right)=0$ provides a simple analytical expression for the spectral location of transmission maximum as a function of $\varepsilon_{1}, \varepsilon_{3}$, and $\lambda_{0}$ :

$$
\lambda_{R}^{(1)}=\frac{\left(\sqrt{\varepsilon_{1}}+\sqrt{\varepsilon_{3}}\right)}{2} \lambda_{0} \quad(h<<\lambda) .
$$

Interestingly, $\lambda_{R}^{(1)}$ does not depend on the slope of $G_{r}$. Equation (23) is in agreement with the values reported in frequency-selective surfaces (FSSs), ${ }^{22,33,34}$ which are designed for operating close to the cutoff wavelength taking strictly $h=$ 0 . However, in Fig. 6 we observe that the linear approximation given by Eq. (23) is valid for $\varepsilon_{3} \leqslant 2$ but strongly deviates from the exact result at larger values of $\varepsilon_{3}$.

We can improve this linear approximation and get some information from fitting both $G^{I}$ and $G^{I I I}$ to a quadratic function close to $\lambda_{0}$ with the form $G_{r}(\lambda ; 1) \approx A\left(\lambda-\lambda_{0}\right)+$ $B\left(\lambda-\lambda_{0}\right)^{2}$. This fitting results in a quadratic equation with the following solution:

$\lambda_{R}^{(2)}=\frac{-p+2 C p \lambda_{0}+\sqrt{\left(p-2 C p \lambda_{0}\right)^{2}-4 C \bar{n}^{2} p\left(C \lambda_{0}^{2}-\lambda_{0}\right)}}{2 C \bar{n}}$, 
where $\bar{n} \equiv\left(\sqrt{\varepsilon_{1}}+\sqrt{\varepsilon_{3}}\right) / 2, \quad p \equiv \sqrt{\varepsilon_{1} \varepsilon_{3}}$, and $C \equiv B / A=$ $\frac{G_{r}{ }^{\prime \prime}\left(\lambda_{0} ; 1\right)}{2 G_{r}{ }^{\prime}\left(\lambda_{0} ; 1\right)}$.

As shown in Fig. $6, \lambda_{R}^{(2)}$ improves the prediction for the spectral position of the maximum transmittance for $\varepsilon_{3} \leqslant 6$. Notice, however, that the range of validity of Eq. (24) is restricted by the requirement that $\lambda_{R}$ must be real, i.e., for $\varepsilon_{3} \leqslant 8$. A simpler expression for $\lambda_{R}$, based on the parabolic dependance of $G_{r}(\lambda ; 1)$, can be obtained by Taylor expanding $\lambda_{R}^{(2)}$ for $C \rightarrow 0$. We get

$$
\lambda_{R}^{(*)}=\frac{\left(\sqrt{\varepsilon_{1}}+\sqrt{\varepsilon_{3}}\right)}{2} \lambda_{0}\left[1-\frac{\left(\sqrt{\varepsilon_{1}}-\sqrt{\varepsilon_{3}}\right)^{2}}{4 \sqrt{\varepsilon_{1} \varepsilon_{3}}}\left(C \lambda_{0}\right)\right] .
$$

Note that, since $C<0$, Eq. (25) predicts larger shifts than Eq. (23). Additionally, $C$ does not depend on either $\varepsilon_{1}$ or $\varepsilon_{3}$ but only on the geometry of the hole.

$\lambda_{R}^{(*)}$ provides a good approximation even for very large values of $\varepsilon_{3}$ (see Fig. 6). This is particularly surprising, as $\lambda_{R}^{(*)}$ is derived from an expression that is not valid for $\varepsilon_{3} \geqslant 8$. So, although the expression for $\lambda_{R}^{(*)}$ is mathematically found for moderate $\varepsilon_{3}$, for larger values of $\varepsilon_{3}$ it must be considered a functional form that happens to fit the numerical results (although without fitting parameters as $\lambda_{0}$ and $C$ can be calculated numerically or extracted from experiments with two different substrates).

To summarize, the whole dependance with $h, \varepsilon_{1}, \varepsilon_{2}$, and $\varepsilon_{3}$ for transmission resonances can be obtained through Eq. (20), whereas Eq. (25) provides the peak position in the limit of thin films.

Regarding intensity, we find that the general expression of transmission at maximum is

$$
T_{R}\left(\lambda_{R} ; \varepsilon_{1}, \varepsilon_{2}, \varepsilon_{3}\right)=\frac{\left|I_{o}\left(\varepsilon_{1}\right)\right|^{2}}{4 \sqrt{\varepsilon_{1}}} \frac{G_{i}^{I I I}\left(\lambda_{R} ; \varepsilon_{1}\right)}{\left[G_{i}^{+}\left(\lambda_{R} ; \varepsilon_{1}, \varepsilon_{3}\right)\right]^{2}},
$$

and following the same steps as those explained for symmetric configurations, we find

$$
T_{R}\left(\lambda_{R} ; \varepsilon_{1}, \varepsilon_{2}, \varepsilon_{3}\right) \propto \frac{\varepsilon_{1}^{1 / 2} \varepsilon_{3}^{3 / 2}}{\left(\varepsilon_{1}^{3 / 2}+\varepsilon_{3}^{3 / 2}\right)^{2}} \lambda_{R}^{2} .
$$

Several regimes can be found for different combinations of substrates and covers in Eq. (27). For instance, in the case of thin films, if $\varepsilon_{1}$ is fixed, this equation predicts a reduction of transmission as the dielectric constant of the substrate increases.

\section{EXTENSION TO HOLE ARRAYS AND OPTICAL REGIME}

In this section we will show how the previous results found for isolated holes can also be applied to hole arrays, and we will also reveal their semiquantitative validity in the optical regime.

Concerning hole arrays in the $\mathrm{THz}$ regime, this theory will be accurate provided that the localized resonance does not interfere with the excitation of surface modes, i.e., $\lambda_{R}>>$ $\sqrt{\varepsilon} p$. When Bragg resonances emerge close to localized resonances, a hybridization of the two resonances occurs, as has been considered in Refs. 11 and 15 .

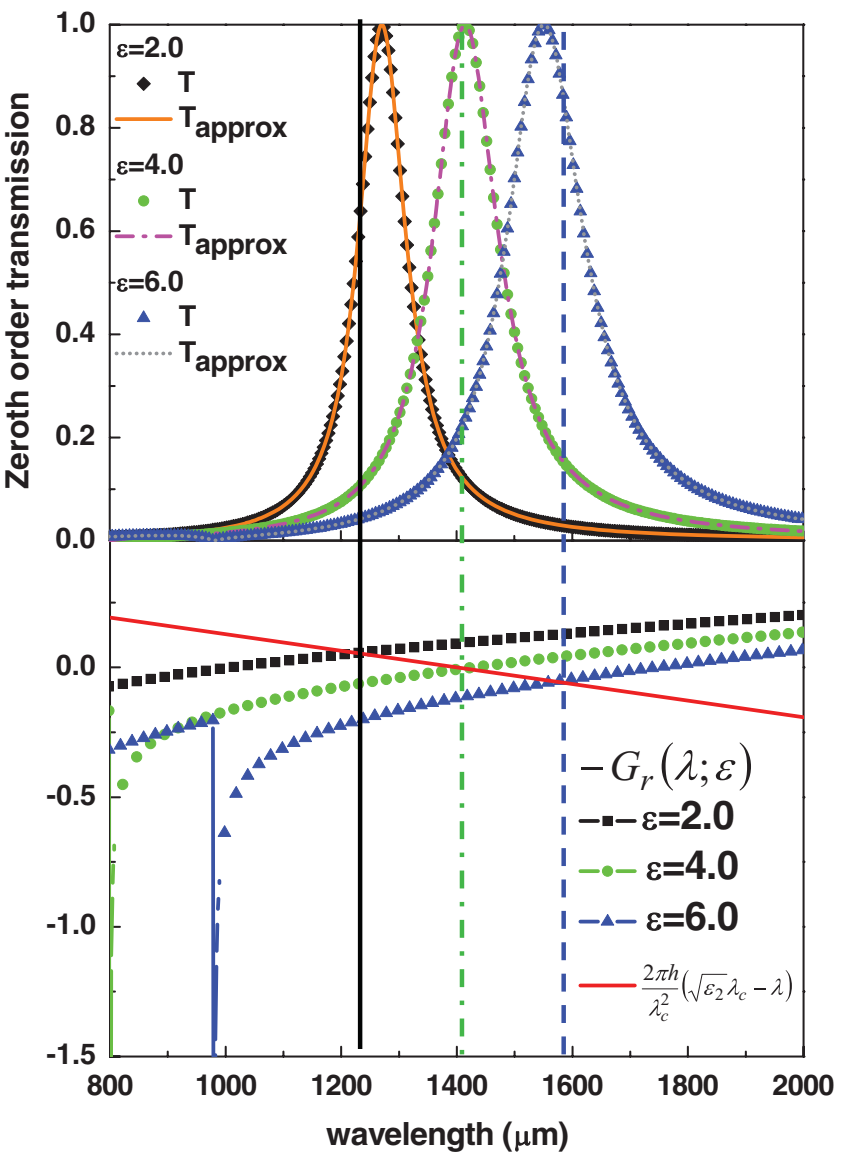

FIG. 7. (Color online) (top) Transmission of light using Eqs. (7) and (13) (see labels) through a hole array $(p=400 \mu \mathrm{m})$ with rectangles $\left(a_{x}=10 \mu \mathrm{m}, a_{y}=350 \mu \mathrm{m}\right)$ drilled in a thin PEC screen of $h=25 \mu \mathrm{m}$ and filled with $\varepsilon_{2}=4$. Symmetric configurations are considered (see labels). (bottom) For the same system as in the top panel, the graphical solution of Eq. (14).

In particular, in Fig. 7 we consider an array $(p=400 \mu \mathrm{m})$ of rectangular holes $\left(a_{x}=10 \mu \mathrm{m}, a_{y}=350 \mu \mathrm{m}\right)$ in a symmetric configuration, drilled in a PEC film of thickness $h=25$ $\mu \mathrm{m}$. In this case we take $\varepsilon_{2}=4$, so the cutoff wavelength is $2 a_{y} \sqrt{\varepsilon_{2}}=1400 \mu \mathrm{m}$. The bottom panel of Fig. 7 shows the graphical solution of Eq. (14) for these hole arrays with $\varepsilon=2,4,6$. Note the presence of divergencies in $G_{r}$, which account for the excitation of surface modes, and that $\lambda_{R} \gg \sqrt{\varepsilon} p$.

The top panel in Fig. 7 shows three different pairs of transmission spectra using either Eq. (7) or Eq. (13). The excellent agreement between pairs of curves confirms the validity of the approximations done to obtain Eq. (13).

Moreover, here it can be seen that the resonant wavelength can be blueshifted or redshifted a factor $\sqrt{\varepsilon / \varepsilon_{2}}$ compared to $\sqrt{\varepsilon_{2}} \lambda_{c}$ as a result of the combination of $\varepsilon_{2}$ and $\varepsilon$. For $\varepsilon<\varepsilon_{2}$, $\lambda_{R}<1400 \mu \mathrm{m}$, and for $\varepsilon>\varepsilon_{2}, \lambda_{R}>1400 \mu \mathrm{m}$.

Regarding the transference of the previous description in the $\mathrm{THz}$ regime to the optical one, we remark that taking into account the finite dielectric constant of metals in the optical regime makes the analytical study with the CMM too complex to find compact expressions for $\lambda_{R}$. 


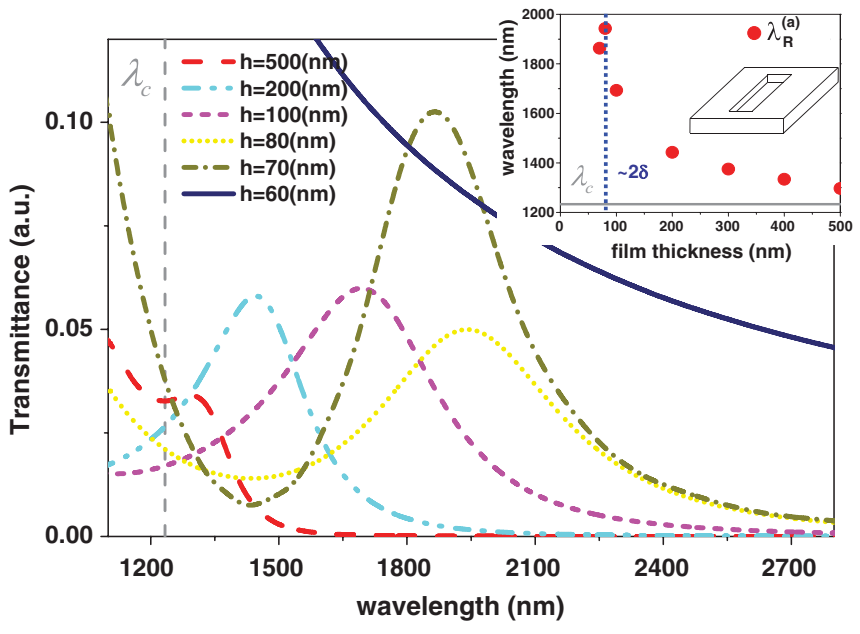

FIG. 8. (Color online) Calculations performed with the FDTD method for the position of transmission maximum as a function of the film thickness in an isolated rectangular hole $\left(a_{x}=50 \mathrm{~nm}\right.$ and $a_{y}=400 \mathrm{~nm}$ ) drilled in a gold film with $\varepsilon_{1}=\varepsilon_{3}=2.25$ and $\varepsilon_{2}=1$. In the inset the vertical blue line depicts $h=2 \delta \sim 60 \mathrm{~nm}$, and the horizontal grey line corresponds to $\lambda_{c}$.

Therefore, as an example and using the FDTD ${ }^{35}$ method, Fig. 8 shows the position of the maximum transmission of a single rectangular hole $\left(a_{x}=50 \mathrm{~nm}, a_{y}=400 \mathrm{~nm}\right)$ drilled in a real metal film (in this case, a gold film) as a function of the film thickness. The dielectric constant of the metal is taken from experimental values tabulated in Refs. 36 and 37 and is conveniently fitted into a Drude-Lorentz model. ${ }^{38,39}$

We observe that, as in the case of PECs, for thick enough films, transmission resonances appear close to the cutoff wavelength, whereas for thin films the peak position appears strongly redshifted compared to $\lambda_{c}$. Similar results have been found in the optical regime also with rectangles in gold $^{21}$ and annular hole arrays in silver, ${ }^{22}$ and this phenomenon is also expected to be found in triangles with acute angles. ${ }^{40}$ Interestingly, in Fig. 8, once $h \approx 2$ skin depths $(\sim 60 \mathrm{~nm})$, the resonant wavelength eventually blueshifts as a result of the typical decaying behavior due to the direct transmission through the metal films. 5,41

\section{CONCLUSIONS}

We have developed a theory for transmission resonances aided by localized modes in the $\mathrm{THz}$ regime for both hole arrays and single holes and for any hole shape of a large-cutoff wavelength. We have shown that the maximum transmission presents large shifts as a function of both the metal thickness and the dielectric constants of the cover and the substrate. This shift becomes also very clear when observing transmission intensities and near-field amplitudes, which are strongly enhanced regarding the free-standing system. We provide analytical expressions for the peak position, which is controlled by the effective admittance of the cover and the substrate as seen by the holes, $G^{I}$ and $G^{I I I}$. We have demonstrated that there are two different length scales related to thick and thin films that control the spectral wavelength of maximum transmission, $\lambda_{c}$ and $\lambda_{0}$, respectively. The first one represents the properties of the waveguides, and it governs transmission resonances in thick films, while the second one characterizes the coupling of the hole to radiative regions, and it rules in thin films.

For symmetric systems $\left(\varepsilon_{1}=\varepsilon_{3}=\varepsilon\right)$, we find that resonances in transmission approximately occur at

$$
\begin{aligned}
& \lambda_{R}^{(a)}=\sqrt{\varepsilon} \lambda_{0} \quad(h \ll \lambda), \\
& \lambda_{R}^{(a)}=\sqrt{\varepsilon_{2}} \lambda_{c} \quad(h \geqslant \lambda / 2),
\end{aligned}
$$

and for asymmetric systems $\left(\varepsilon_{1} \neq \varepsilon_{3}\right)$ they occur at

$\lambda_{R}^{(a)}=\frac{\left(\sqrt{\varepsilon_{1}}+\sqrt{\varepsilon_{3}}\right)}{2} \lambda_{0}\left[1-\frac{\left(\sqrt{\varepsilon_{1}}-\sqrt{\varepsilon_{3}}\right)^{2}}{4 \sqrt{\varepsilon_{1} \varepsilon_{3}}}\left(C \lambda_{0}\right)\right](h \ll \lambda)$, $\lambda_{R}^{(a)}=\sqrt{\varepsilon_{2}} \lambda_{c} \quad(h \geqslant \lambda / 2)$,

with $C$ being a fitting constant that depends on the hole shape. Transmission intensities have been also discussed in both symmetric and asymmetric configurations. Finally, we have shown that our predictions are quantitatively valid for hole arrays (provided $\lambda_{R}>>\sqrt{\varepsilon} p$ ) and qualitatively valid in the optical regime.

\section{ACKNOWLEDGMENTS}

We acknowledge support from the Spanish Ministry of Science and Innovation (Projects MAT2008-06609-C02 and CSD2007-046-Nanolight.es).
${ }^{1}$ T. W. Ebbesen, H. L. Lezec, H. F. Ghaemi, T. Thio, and P. A. Wolff, Nature (London) 391, 667 (1998).

${ }^{2}$ F. J. Garcia-Vidal, L. Martin-Moreno, T. W. Ebbesen, and L. Kuipers, Rev. Mod. Phys. 82, 729 (2010).

${ }^{3}$ L. Martín-Moreno, F. J. García-Vidal, H. J. Lezec, K. M. Pellerin, T. Thio, J. B. Pendry, and T. W. Ebbesen, Phys. Rev. Lett. 86, 1114 (2001).

${ }^{4}$ A. Azad and W. Zhang, Opt. Lett. 30, 2945 (2005).

${ }^{5}$ S. G. Rodrigo, L. Martín-Moreno, A. Y. Nikitin, A. V. Kats, I. S. Spevak, and F. J. García-Vidal, Opt. Lett. 34, 4 (2009).

${ }^{6}$ K. J. Klein Koerkamp, S. Enoch, F. B. Segerink, N. F. van Hulst, and L. Kuipers, Phys. Rev. Lett. 92, 183901 (2004).
${ }^{7}$ R. Gordon, A. G. Brolo, A. McKinnon, A. Rajora, B. Leathem, and K. L. Kavanagh, Phys. Rev. Lett. 92, 037401 (2004).

${ }^{8}$ H. Cao and A. Nahata, Opt. Express 12, 3664 (2004).

${ }^{9}$ A. Degiron, H. J. Lezec, N. Yamamoto, and T. W. Ebbesen, Opt. Commun. 239, 61 (2004).

${ }^{10}$ K. L. van der Molen, K. J. Klein Koerkamp, S. Enoch, F. B. Segerink, N. F. van Hulst, and L. Kuipers, Phys. Rev. B 72, 045421 (2005).

${ }^{11}$ A. Degiron and T. W. Ebbesen, J. Opt. A 7, S90 (2005).

${ }^{12}$ F. J. García-Vidal, E. Moreno, J. A. Porto, and L. Martín-Moreno, Phys. Rev. Lett. 95, 103901 (2005).

${ }^{13}$ Z. Ruan and M. Qiu, Phys. Rev. Lett. 96, 233901 (2006). 
${ }^{14}$ F. J. García-Vidal, L. Martín-Moreno, E. Moreno, L. K. S. Kumar, and R. Gordon, Phys. Rev. B 74, 153411 (2006).

${ }^{15}$ A. Mary, S. G. Rodrigo, L. Martín-Moreno, and F. J. García-Vidal, Phys. Rev. B 76, 195414 (2007).

${ }^{16}$ F. Medina, J. A. Ruiz-Cruz, F. Mesa, J. M. Rebollar, J. R. MontejoGarai, and R. Marqués, Appl. Phys. Lett. 95, 071102 (2009).

${ }^{17}$ A. J. L. Adam, J. M. Brok, M. A. Seo, K. J. Ahn, D. S. Kim, J. H. Kang, Q. H. Park, M. Nagel, and P. C. Planken, Opt. Express 16, 7407 (2008).

${ }^{18}$ L. Guestin, A. J. L. Adam, J. R. Knab, M. Nagel, and P. C. M. Planken, Opt. Express 17, 17412 (2009).

${ }^{19}$ D. J. Park, S. B. Choi, Y. H. Ahn, F. Rotermund, I. B. Sohn, C. Kang, M. S. Jeong, and D. S. Kim, Opt. Express 17, 12493 (2009).

${ }^{20}$ M. A. Seo, A. J. L. Adam, J. H. Kang, J. W. Lee, K. J. Ahn, Q. H. Park, P. C. M. Planken, and D. S. Kim, Opt. Express 16, 20484 (2008).

${ }^{21}$ J. H. Kang, J.-H. Choe, D. S. Kim, and Q.-H. Park, Opt. Express 17, 15652 (2009).

${ }^{22}$ A. Roberts and L. Lin, Opt. Mater. Express 1, 480 (2011).

${ }^{23}$ F. J. García de Abajo, Opt. Express 10, 1475 (2002).

${ }^{24}$ J. Bravo-Abad, L. Martín-Moreno, and F. J. García-Vidal, Phys. Rev. E 69, 026601 (2004).

${ }^{25}$ A. Roberts and S. M. Orbons, IEEE Trans. Antennas Propag. 36, 607 (1988).

${ }^{26}$ F. de León-Pérez, G. Brucoli, F. J. García-Vidal, and L. MartínMoreno, New J. Phys. 10, 105017 (2008).

${ }^{27}$ A. Y. Nikitin, D. Zueco, F. J. García-Vidal, and L. Martín-Moreno, Phys. Rev. B 78, 165429 (2008)

${ }^{28}$ J. Bravo-Abad, F. J. García-Vidal, and L. Martín-Moreno, Phys. Rev. Lett. 93, 227401 (2004).
${ }^{29}$ Equation (2) provides the continuity of the magnetic field, which is an axial vector. $E$ and $E^{\prime}$ have opposite signs, so if the system is illuminated from region III, Eq. (1) is still valid and the illumination would still be represented by a positive $I_{0}$.

${ }^{30}$ M. J. Lockyear, A. P. Hibbins, J. R. Sambles, and C. R. Lawrence, Phys. Rev. Lett. 94, 193902 (2005).

${ }^{31}$ F. I. Baida, A. Belkhir, D. Van Labeke, and O. Lamrous, Phys. Rev. B 74, 205419 (2006).

${ }^{32}$ S. M. Orbons and A. Roberts, Opt. Express 14, 12623 (2006).

${ }^{33}$ R. C. Compton, L. B. Whitbourn, and R. C. McPhedran, Appl. Opt. 23, 3236 (1984)

${ }^{34}$ B. A. Munk, Frequency Selective Surfaces: Theory and Design (Wiley, New York, 2000).

${ }^{35} \mathrm{~A}$. Taflove and S. Hagness, Computational Electrodynamics: The Finite-Difference Time-Domain Method (Artech House, Boston, 2000).

${ }^{36}$ Handbook of Optical Constants of Solids, Academic Press Handbook Series, edited by E. D. Palik (Academic, New York, 1985).

${ }^{37}$ Handbook of Optical Constants of Solids II, edited by E. D. Palik (Academic, Boston, 1991).

${ }^{38}$ S. G. Rodrigo, F. J. García-Vidal, and L. Martín-Moreno, Phys. Rev. B 77, 075401 (2008).

${ }^{39}$ F. Hao and P. Nordlander, Chem. Phys. Lett. 446, 115 (2007).

${ }^{40}$ S. G. Rodrigo, O. Mahboub, A. Degiron, C. Genet, F. J. GarcíaVidal, L. Martín-Moreno, and T. W. Ebbesen, Opt. Express 18, 23691 (2010).

${ }^{41}$ T. Rindzevicius, Y. Alaverdyan, B. Sepulveda, T. Pakizeh, M. Käll, R. Hillenbrand, J. Aizpurua, and F. J. García de Abajo, J. Phys. Chem. C 111, 1207 (2007). 